\title{
Application of an Ageing Model to a Hydrogen- Fuelled PEM Fuel Cell
}

\author{
Matteo Riccardi ${ }^{1, a)}$, Marcello Romagnoli ${ }^{1)}$, and Giuseppe Corda ${ }^{1)}$, \\ ${ }^{1}$ Department of Engineering "Enzo Ferrari”, University of Modena and Reggio Emilia, Via Vivarelli \\ 10, Modena 41125, Italy
}

\begin{abstract}
Hydrogen is one of the most promising energy vectors for achieving the decarbonization of the propulsion systems market. Polymer Electrolyte Membrane Fuel Cell system (PEMFCs) stand out in this panorama, thanks to reduced activation times and low temperatures of use, easily adapting to the needs of the sector. In this paper, a multi-phase, multicomponent and non-isothermal 3D-CFD model is proposed to simulate the effects of PEMFC ageing, limiting the system durability due to slow membrane corrosion and loss of material performance and properties degradation, resulting in a reduced current density under equal voltage.

The model is applied both in 1D and 3D frameworks implementing a validated model from literature, allowing to estimate the useful life of the cell as a function of parameters such as the degradation rate and the crossover rate. Simulations are carried out at different membrane thicknesses and for different membrane conductivities, using a hydrogenfuelled serpentine-type PEM fuel cell. The study relevance lies in the possibility to investigate the critical aspects limiting PEMFC system durability, as well as the optimal conditions of use, and it allows to identify corrective design actions.
\end{abstract}

\section{Introduction}

Increasingly stringent emissions regulations are forcing a drastic change in the transportation sector. Internal combustion vehicles (ICEVs) are thus undergoing continuous complexity increase and numerical investigations [1-4], in order to accommodate convenient solutions to contain emissions (particulate filters, catalytic reduction systems) or to combine with electric motors to create hybrid solutions. In this scenario, the transition to a new energy vector seems increasingly likely in the near future. The most plausible one seems to be hydrogen, either used as a fuel in internal combustion engines or to power solutions such as polymer electrolyte membrane fuel cells (PEMFC). The latter system allows the direct generation of electricity, with water and heat as the only products of the process. Hydrogen will play a fundamental role in an increasingly cutting-edge panorama of energy conversion systems [5-8] and thus of simulation techniques [9,10]. Among the pioneering models proposed in the literature, the 1D models by Springer et al [11] and Bernardi et al. [12] paved the way for membrane models, including a detailed water uptake curve.

\footnotetext{
*Corresponding author: matteo.riccardi@unimore.it
} 
Moving to 3D models, a key aspect in PEMFC modelling is the grid resolution, especially in the through-plane direction (i.e. along the cell thickness) where higher gradients (e.g. species concentration, thermal and electric fields) develop despite small length scales (typically 10$1000 \mu \mathrm{m})[13]$. In the proposed model the fundamental and technology-limiting issue of cell ageing is analyzed. Relevant studies from literature deal with this topic, e.g. the work of Yousfi-Steiner et al. [14] or Ramaswamy et al. [15]. In [16] Karpenko-Jereb et al. proposed a semi-empirical model where the degradation rates are a function of the cell crossover rate and changes in the physical-chemical properties of the polymer electrolyte membrane operating in the cell are also considered. The degradation processes in polymeric electrolyte membranes can be of three types: 1) mechanical destruction, causing pinholes and cracks to form in the polymer; 2) chemical degradation; 3) thermal degradation, occurring at high temperature $\left(>150^{\circ} \mathrm{C}\right)$ and leading to the membrane drying. Using the results from the 1D version of the model, a 3D version of the same is proposed and implemented to understand the reasons for performance degradation with time. The membrane degradation model is coupled with the CFD model and applied to analyze the behavior of the cell as a function of time. Operating conditions have a marked influence on the membrane degradation rate and on its non-uniformity over the membrane surface.

\section{Numerical Model}

In this study, a multi-phase, multi-physics modelling approach [17] is adopted to consider the presence of multi-phase fluids (e.g., liquid/vapor) in a PEM fuel cell, as well as multiple components (fluid and solid). This introduces modelling challenges due to simultaneous physical and electrochemical processes (fluid, heat, and charge transport) and phase change. These processes are simulated by solving conservation equations for mass, momentum, energy, chemical species, and electric charges (electrons and protons). The presence of porous media imposes a dedicated flow treatment, since modelling the transport of reactants and the removal of products through the gas diffusion layers is crucial for a correct prediction of the cell performance. In addition, a multiphase fluid treatment is required for the possible presence of liquid water at the cathode due to the low pressure and temperature conditions present. Finally, an important role is played by the characterization of the electrolyte, which is modelled as impermeable to fluids, but which allows the transport of ions and water molecules via electro-osmotic drag effect. With reference to a preliminary validation of the model in [18], the CFD-3D model is here developed extensively as reported in [19]. The multiphase approach adopted in this study is the mixture multiphase (MMP) approach. This method assumes that the two fluid phases are miscible and at equilibrium, and their motion can be simulated as that of a unique continuum. This corresponds to a Eulerian representation of low-saturated streams where isolated liquid droplets are transported by the bulk flow. This simplification implies that a single continuity, momentum, and energy equation is solved for the eulerian mixture, and the phases subdivision (sharing the same mixture velocity, $u_{\text {mix }}$ ) is handled by a dedicated transport equation for the volume fraction and the phase relative velocity, or postprocessed via thermodynamic values [20]. 


\section{Governing equations}

The transport equations are given below assuming the steady-state case, i.e. where the term $\frac{\partial}{\partial t}=0$.

Continuity: $\quad \nabla\left(\rho_{\text {mix }} \vec{u}_{\text {mix }}\right)=S_{m}$

Momentum: $\nabla\left(\frac{\rho_{m i x} \vec{u}_{m i x} \vec{u}_{m i x}}{\varepsilon^{2}}\right)=-\nabla p+\mu \nabla\left[\nabla\left(\frac{\vec{u}_{m i x}}{\varepsilon}\right)+\nabla\left(\frac{\vec{u}_{m i x}^{T}}{\varepsilon}\right)\right]-\frac{2}{3} \mu \nabla\left[\nabla\left(\frac{\vec{u}_{m i x}}{\varepsilon}\right)\right]+\mathrm{S}_{\mathrm{u}}$

Species:

$$
\nabla\left(\rho_{\text {mix }} Y_{i} \vec{u}_{m i x}\right)=\nabla\left(\rho D_{i}^{e f f} \nabla Y_{\mathrm{i}}\right)+\mathrm{S}_{\mathrm{i}}
$$

Energy:

$$
\nabla\left[\left(\rho_{\text {mix }} c_{p}\right)^{e f f} \vec{u}_{m i x} T\right]=\nabla\left(k^{e f f} \nabla T\right)+S_{T}
$$

Charge:

$$
\begin{aligned}
& \nabla\left(\kappa^{e f f} \nabla \varphi_{s}\right)+S_{\varphi_{s}}=0 \\
& \nabla\left(\sigma^{e f f} \nabla \varphi_{e}\right)+S_{\varphi_{e}}=0
\end{aligned}
$$

\section{Ageing Model}

With reference to the work of Karpenko-Jereb [16], the polymer degradation kinetics depend on the concentration of hydroxyl radicals in the membrane, which is related to the oxygen concentration. Since at the operating condition considered $\left(\mathrm{p}_{\mathrm{c}}=\mathrm{p}_{\mathrm{a}}=1\right.$ bar $)$, the oxygen concentration is determined by the diffusion of $\mathrm{O}_{2}$. Therefore, the following correlation between the membrane degradation rate and the $\mathrm{O}_{2}$ crossover rate is proposed:

$$
r^{\text {curr }}=r^{\text {ref }} \cdot \frac{J_{O 2}^{\text {curr }}}{J_{O 2}^{\text {ref }}}
$$

where $r^{r e f}[1 / \mathrm{h}]$ and $J_{O 2}^{r e f}\left[\mathrm{~mol} /\left(\mathrm{cm}^{2} \cdot s\right)\right]$ are the reference degradation rate and oxygen flux crossover rate through the membrane at the reference operating conditions, while $r^{\text {curr }}, J_{O 2}^{\text {curr }}$ are the degradation rate and oxygen flux crossover rate through the membrane at the current operating conditions. To simplify the calculation of the ratio of $\mathrm{O}_{2}$ crossover fluxes under two different operating conditions, we use $\mathrm{Eq}(8)$ to fit the experimental data of $\mathrm{H}_{2}$ crossover rate as a function of pressure gradient, temperature, relative humidity $(\mathrm{RH})$, and membrane thickness. The calculations were performed on different membrane thicknesses derived from the 1D model. Using multiple linear regression to analyze the data obtained, the following expression is obtained:

$$
J_{H_{2}}=\left[a_{0}+a_{1} \cdot T_{\left[{ }^{\circ} C\right]}^{\text {curr }}+a_{2} \cdot R H_{[\%]}^{\text {curr }}+a_{3} \cdot p_{[\text {bar }]}^{\text {curr }}+\frac{a_{4}}{\ln \left(L_{\text {mem }}^{\text {curr }}\right)}\right]
$$

where $a_{0}=-2.6492 ; a_{1}=0.0180 ; a_{2}=0.0036 ; a_{3}=0.5992 ; a_{4}=10.840$. In this expression $\mathrm{p}$ is the $\mathrm{H}_{2}$ pressure at the anode, $L_{\text {mem }}=[\mathrm{mm}], \mathrm{J}_{\mathrm{H} 2}=\left[\mathrm{mol} / \mathrm{cm}^{2} \cdot \mathrm{s}\right]$. As shown in Refs. [21-23], the ratio of $\mathrm{O}_{2}$ to $\mathrm{H}_{2}$ crossover flux through Nafion membranes does not depend on environmental conditions such as T, p, RH and can be considered as a constant value, i.e., $J_{\text {curr } O 2}=J_{\text {ref }} \mathrm{O}_{2}=J_{\text {curr }_{\mathrm{H} 2}}=J_{\text {ref }_{\mathrm{H} 2}}=$ cost. In the absence of the gas pressure gradient across the membrane ( $a \mathrm{p}_{\mathrm{c}}=\mathrm{p}_{\mathrm{a}}=1$ bar), the following relationship is used: 


$$
\frac{\mathrm{JO}_{2}{ }^{\text {curr }}}{\mathrm{JO}_{2}{ }^{\text {ref }}}=\frac{\left[-2.6492+0.0180 \cdot T_{\left[{ }^{\circ} \mathrm{C}\right]}^{\text {curr }}+0.0036 \cdot R H_{[\%]}^{\text {curr }}+0.5992 \cdot p_{[\text {bar }]}^{\text {curr }}+\frac{10.840}{\ln \left(L_{\text {mem }}^{\text {curr }}\right)}\right]}{\left[-2.6492+0.0180 \cdot T_{\left[{ }^{\circ} \mathrm{C}\right]}^{r e f}+0.0036 \cdot R H_{[\%]}^{r e f}+0.5992 \cdot p_{[\text {bar }]}^{\text {ref }}+\frac{10.840}{\ln \left(L_{\text {mem }}^{r e f}\right)}\right]}
$$

It should be noted that we use Eq. (9) to calculate the degradation rate of the membrane thickness and the membrane conductivity at open circuit potential. In fact, the degradation rate is also dependent on the operating voltage, finally leading to the linear fitting in Eq. 10, valid for $0.4-1.2 \mathrm{~V}$ :

$$
r J=\frac{J_{O_{2}}{ }^{c u r r}(U)}{J_{O_{2}}{ }^{r e f}}=(1.12 \cdot V-0.06)
$$

These are fully implemented in the 1D model which processes the results for different voltage values for the PEMFC. The degradation rates of membrane thickness and conductivity used as input data in the semi-empirical model of membrane degradation are listed below. Degradation rates are estimated from experimental data reported by Yuan et al. $[18,19]$.

$$
\begin{aligned}
& r_{L_{m e m}}^{\text {ref }}=3.71 \cdot 10^{-4} 1 / h \rightarrow[24] \text { Used to calculate } r_{L_{m e m}}^{\text {curr }}(V) 1 / h \\
& r_{\sigma_{m e m}}^{\text {ref }}=5.64 \cdot 10^{-4} 1 / h \rightarrow[25] \text { Used to calculate } r_{\sigma_{m e m}}^{\text {curr }}(V) 1 / h
\end{aligned}
$$

Considering the previous equations, the calculation of the membrane degradation rate becomes:

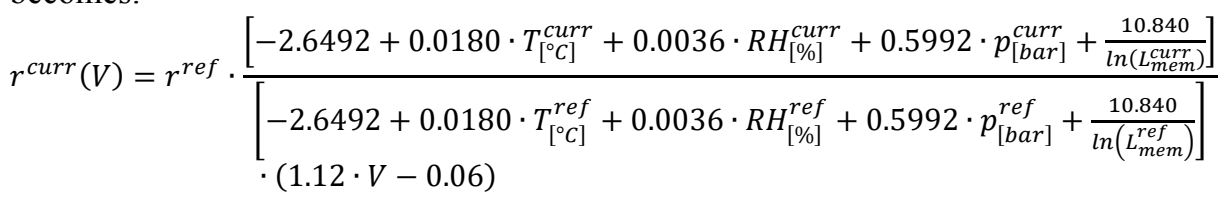

Based on the value of the degradation rate $r_{c u r r}(\mathrm{~V})$, the thickness of the membrane and the conductivity in the degraded condition are calculated by the following equations:

$$
\begin{aligned}
& L_{\text {mem }}(t)=L_{\text {mem }}^{\text {BoL }} \cdot\left[1-t \cdot r_{L_{m e m}}^{\text {curr }}(V)\right] \\
& \sigma_{\text {mem }}(t)=\sigma_{\text {mem }}^{\text {BoL }} \cdot\left[1-t \cdot r_{\sigma_{\text {mem }}}^{\text {curr }}(V)\right]
\end{aligned}
$$

These data are implemented in the 3D model allowing to have a simulated aged cell 3D state after a given lifetime of interest, while maintaining the calculation in steady-state conditions.

\section{CFD model}

The 3D model for CFD simulations is created using SIMCENTER STAR-CCM+ 2020.3 provided by SIEMENS DISW, and simulations are run on a 16 CPU Linux workstation. This PEM fuel cell model was also numerically studied in $[18,19]$. The characterization of the gas flow at anodic and cathodic channels reproduces the conditions of the laboratory tests, where the cell was supplied with hydrogen and air flows, both humidified with a relative humidity of $100 \%$. The multi-component mixture properties are summarized in Table 2, while Table 3 reports the constants used to model the electrochemical reactions. A three-dimensional model of the tested PEM fuel cell is created for CFD simulations. The discretized grid 


\begin{tabular}{clcc}
\hline Physics & \multicolumn{1}{c}{ Property } & Value & Reference \\
\hline \multirow{2}{*}{ Anode } & Exchange Current Density $\left[\mathrm{A} \mathrm{cm}^{-2}\right]$ & $250 \times 10^{-4}$ & \\
& Apparent Charge Transfer Coefficient & $\alpha_{a}=2 ; \alpha_{c}=2$ & {$[26]$} \\
\multirow{2}{*}{ Cathode } & Exchange Current Density $\left[\mathrm{A} \mathrm{cm}^{-2}\right]$ & $0.25 \times 10^{-4}$ & \\
& Apparent Charge Transfer Coefficient & $\alpha_{a}=2 ; \alpha_{c}=2$ & {$[26]$} \\
\hline
\end{tabular}

consists in approximately $1 \mathrm{M}$ finite volume cells. In Figure 1 the structured mesh for the anode gas channel is shown.

Table 1. Parameters of the electrochemical reactions.

Table 2. Physical properties of cell's main components.

\begin{tabular}{clcc}
\hline Domain & \multicolumn{1}{c}{ Property } & Value & Reference \\
\hline \multirow{2}{*}{ Anode } & Relative Humidity [\%] & & $100 \%$ \\
& & & \\
& Molecular Diffusivity $\left[\mathrm{m}^{2} \mathrm{~s}^{-1}\right]$ & $D_{\mathrm{H} 2}=1.1 \times 10^{-4} ; D_{\mathrm{H} 2 \mathrm{O}}=7.35 \times 10^{-5}$ & \\
& Relative Humidity [\%] & $100 \%$ & \\
\multirow{2}{*}{ Cathode } & & $27]$ \\
& Molecular Diffusivity $\left[\mathrm{m}^{2} \mathrm{~s}^{-1}\right]$ & $D_{\mathrm{O} 2}=3.3 \times 10^{-5} ; D_{\mathrm{N2}}=4 \times 10^{-5}$ & \\
\hline
\end{tabular}

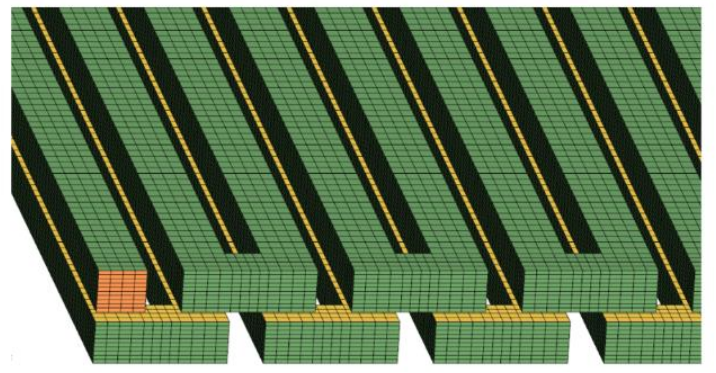

Figure 1. Mesh distribution in the anode and cathode gas channel

The grid creation has been carried out using the Direct Mesh technique both for the channels and for the remaining parts (GDL, membrane, bipolar plates). Once the patch has been defined, it is possible to manage the volumetric distribution of the surface mesh along a guide surface, defining the number of cell layers and the parameters of a possible non-homogeneous distribution and using elongation functions. In this way a structured three-dimensional mesh is obtained, such as the one in Figure 1. The Direct Mesh technique allows to have an excellent grid density control and the conformality of the interfaces between the various components, thus ensuring computational efficiency and stability. Due to the ageing phenomena, the membrane thickness undergoes a reduction due to material removal, as reported in Figure 2. This parameter obtained from the 1D model is then implemented in the geometry of the 3D CFD model to simulate a $2000 \mathrm{~h}$ aged state, and simulations reach convergence on average around 1000 iterations for the various operating voltages. 


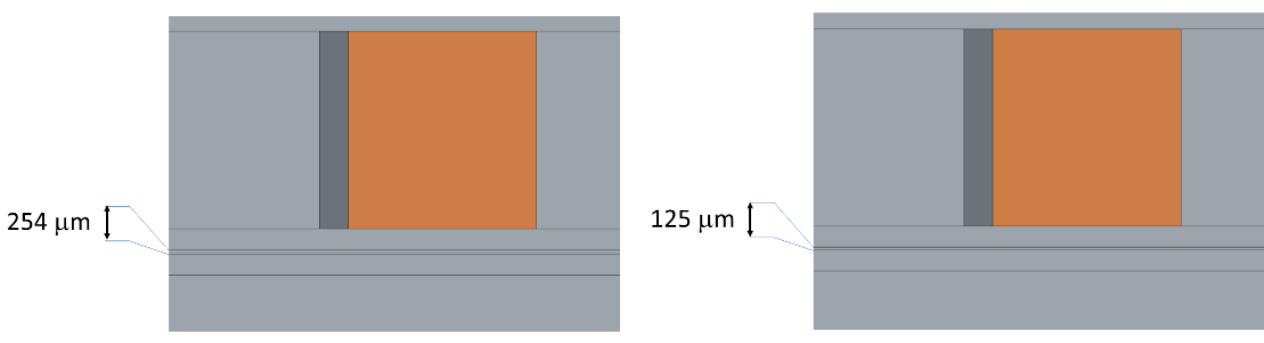

Figure 2. Begin of life (BoL) thickness of membrane (left) and reduction of thickness in membrane after $2000 \mathrm{~h}$ of ageing for CFD model (right).

\section{Results}

Different voltages were analyzed for the cell. The prediction of an aged condition requires not only a future time, but also an operating voltage up to that time (assuming steady-state operation). This is because the ageing rate depends on the voltage itself. In this paper the results at $0.5 \mathrm{~V}$ are shown. Comparing the results for the degradation rates of membrane thickness and ionic conductivity, we note that the latter is always higher, and conductivity reaches an unacceptable value before the membrane thickness degrades to a critical value. This negatively affects the cell performance and indicated the ions conduction through the membrane as the dominant ageing factor. This is verified for all voltages and it is due to the different degradation rates and variable values at begin of life $(\mathrm{BoL})$. This follows from Eq. 14 and Eq. 15, as the thickness and conductivity values $L_{\text {mem }}(t)$ and $\sigma_{m e m}(t)$ are directly related to $r^{\text {curr }}(\mathrm{V})$, ultimately depending on the operating voltage. The modification of the two variables $\left(L_{m e m}\right.$ and $\left.\sigma_{m e m}\right)$ as a function of both time and operating voltage is illustrated in Figure 4.

\section{Degradation Rate}

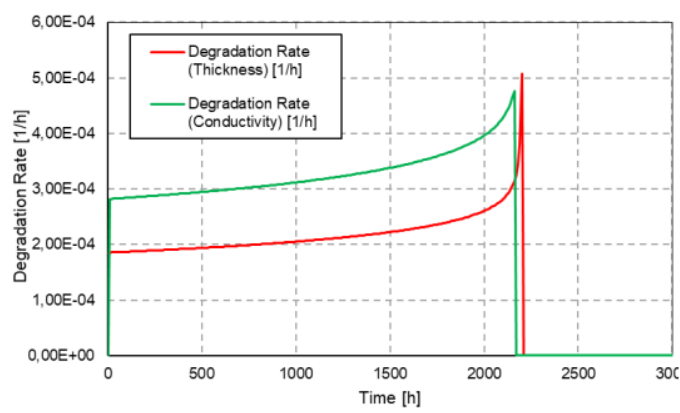

\section{Membrane Thickness / Conductivity}

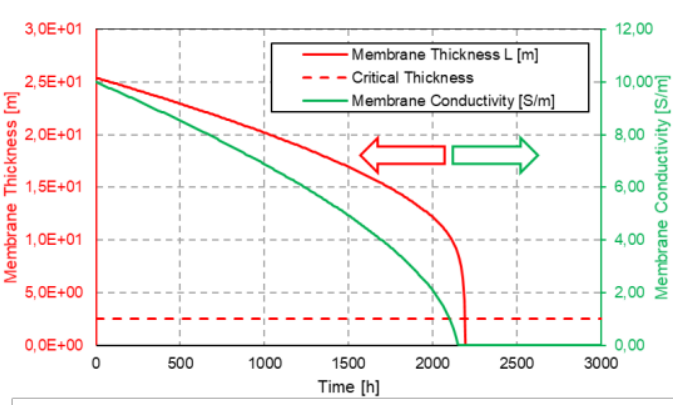

Figure 3. Degradation rate for membrane thickness and ionic conductivity (left); values for membrane and ionic conductivity (right) caused by ageing. 

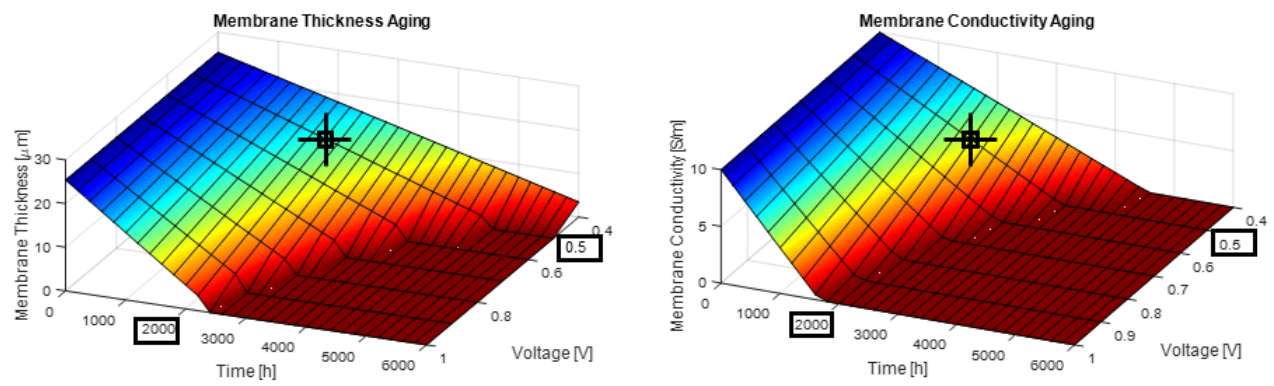

Figure 4. Degradation of membrane thickness (left) and conductivity (right) by ageing, with indication of the 2000 h operation at $0.5 \mathrm{~V}$.
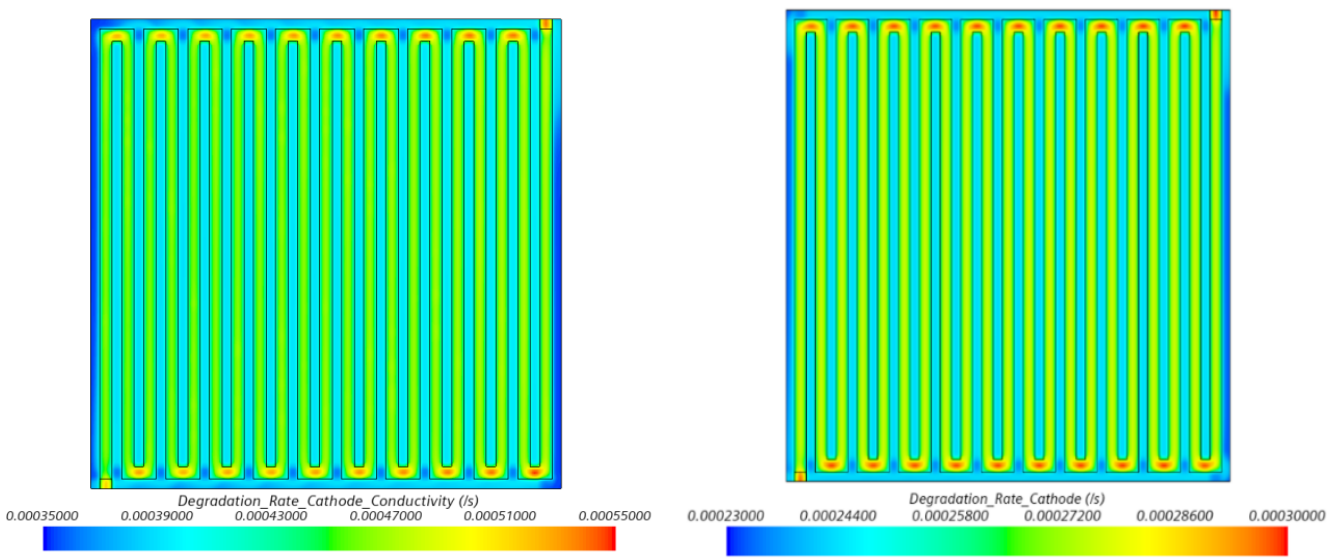

Moving to a 3D analysis of the degradation rates, it is possible to identify the regions most subject to ageing effect and the reasons for that. The degradation rate for both thickness and conductivity reaches a maximum in the outer area of the cell, particularly in the center of the serpentine curves and along the gas channel axis were the electric potential (V) has the highest value. Figure 5 shows the field at the interface between the membrane and the cathode-side GDL for the 3D model at convergence, and we note that the degradation rate of conductivity is higher than the thickness degradation rate. As mentioned earlier, this further confirms the fact that it is $\sigma_{m e m}(t)$ that is most subject to degradation by ageing, thus representing the most critical factor.

Figure 5. Degradation rate for thickness (left) and degradation rate conductivity (right) for cathode side.

The polarization curves in Figure 6 represent the comparison between the cell at begin of life and the cell subject to degradation after $2000 \mathrm{~h}$ of use at $0.5 \mathrm{~V}$. It is interesting to note that the slope of the polarization curve for the aged cell state slightly increases, indicating higher Ohmic losses induced by ageing. This is a critical issue especially at high current density operations, reducing the net output power produced by the cell. The ageing process acts via a combined degradation of the membrane thickness and of its ionic conductivity; however, the two effects partially offset each other, as a membrane thickness reduction alone would reduce Ohmic overpotential, whereas lower ionic conductivity clearly increases it. This explains the relatively similar polarization curves in Figure 6 despite the largely different 
values predicted by the model and reported in Figure 3, and it confirms the investigation possibilities given by advanced CFD models applied to PEMFC systems.

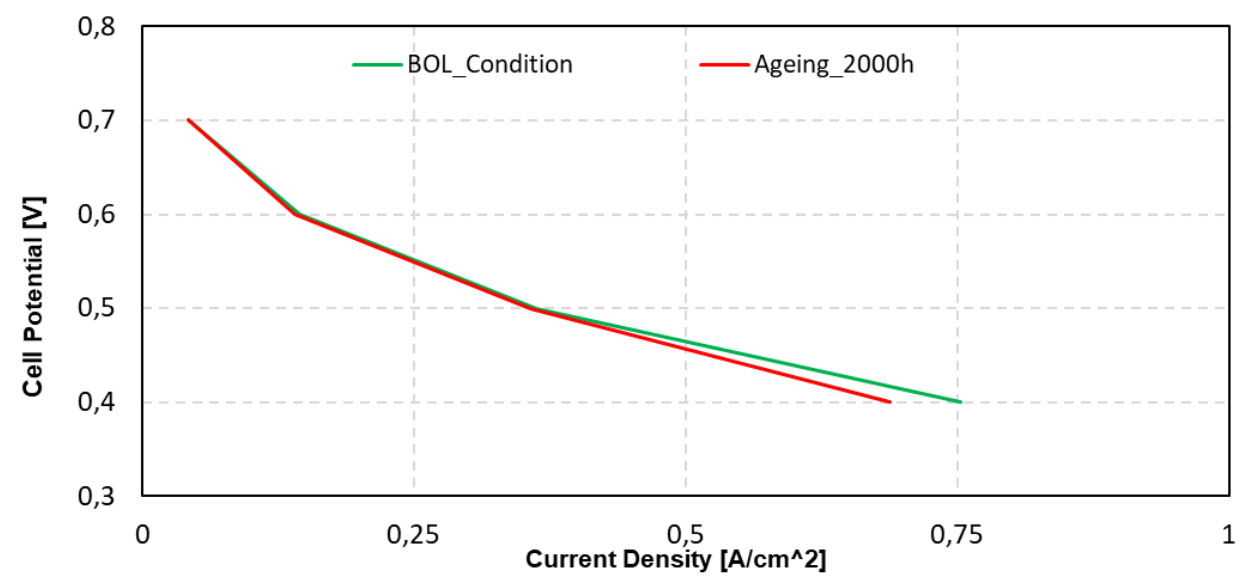

Figure 6. Polarization curve and the effect of ageing after $2000 \mathrm{~h}$.

\section{Conclusions}

The paper describes the development of a degradation model to simulate the performance degradation of a polymer electrolyte fuel cell (PEMFC) as a function of time. The model consists of two main parts: a semi-empirical 1D degradation model, which is coupled with a 3D-CFD multi-phase model. Multi-dimensional CFD analyses of the performance of a single cell show that the decrease in the membrane conductivity is not uniform and it depends on the local operating conditions. In addition, the degradation model is applied to simulate the evolution of the cell current density at a given cell voltage. The estimated degradation in the material properties is analyzed and explanations for it are given. Moreover, the combined (opposed) effect of membrane erosion and conductivity reduction is discussed, explaining the apparently similar cell voltage-current output under largely different material properties. The study demonstrates the understanding possibilities given by the application of advanced numerical models to PEMFC, with regard to the crucial issue of ageing, and it shows how the ageing process can be considered at the design stage of PEMFC systems.

\section{References}

1. A. d'Adamo et al., SAE Int. J. Engines 12(1):45-56, 2019, https://doi.org/10.4271/03-12-01-0007

2. A. d'Adamo et al., SAE Int. J. Engines 9(1):618-630, 2016, doi:10.4271/2016-010581

3. A. d'Adamo et al., Applied Energy Volume 249, 1 September 2019, Pages 126-142, https://doi.org/10.1016/j.apenergy.2019.04.093 
4. S. Fontanesi et al., SAE Technical Paper 2013-24-0031, https://doi.org/10.4271/2013-24-0031

5. N. Zarei et al., Energy Sources, Part A: Recovery, Utilization, and Environmental Effects Volume 42, 2020 - Issue 1, doi: 10.1080/15567036.2019.1587057

6. S.Y. Dibazar et al., Processes 2020, 8, 586; doi:10.3390/pr8050586

7. M.A. Ehyaei et al., Processes 2020, 8, 1277; doi:10.3390/pr8101277

8. S. Esfandi, Energies 2020, 13, 6453; doi:10.3390/en13236453

9. K. Valizadeh, Energy Sci Eng. 2019;00:1-16. DOI: 10.1002/ese3.514

10. A. Davarpanah et al., Energy Sources, Part A: Recovery, Utilization, and Environmental Effects, DOI: 10.1080/15567036.2018.1549133

11. T.E. Springer et al., Journal of Electrochemical Society 138(8, 2342):2334, 1991, doi:10.1149/1.2085971.

12. D.M. Bernardi, J. Electrochem. Soc. 1992, 139, 2477.

13. A. D'Adamo et al., SAE Technical Paper 2020-24-0016, 2020, https://doi.org/10.4271/2020-24-0016

14. N. Yousfi-Steiner et al., Journal of power sources 183.1 (2008): 260-274.

15. N. Ramaswamy et al., Electrochimica Acta 53.8 (2008): 3279-3295.

16. L. Karpenko-Jereb et al., International Journal of Hydrogen Energy, https://doi.org/10.1016/j.ijhydene.2016.05.229.

17. C-Y. Wang, Chem. Rev. 2004, 104, 4727-4766, doi: 10.1021/cr020718s.

18. M. Riccardi et al., E3S Web Conf. Volume 197, 2020, doi: https://doi.org/10.1051/e3sconf/202019705004

19. A. d'Adamo et al., Processes 2021, 9(3), 564; https://doi.org/10.3390/pr9030564

20. A. d'Adamo, Processes 2021, 9(4), 688; https://doi.org/10.3390/pr9040688M.

21. Schalenbach et al., Meas J Phys Chem C 2015;119:25145e55.

22. K. Broka., J Appl Electrochem 1997;27:117e24.

23. P. Gode et al., J Electroanal Chem 2002;518:115e22

24. X. Yuan, J Power Sources 2010;195:7594e9.

25. X. Yuan et al., J Power Sources 2012;205:324e34.

26. J. Macedo-Valencia et al., International Journal of Hydrogen Energy 41 (2016) 23425-23433, http://dx.doi.org/10.1016/j.ijhydene.2016.10.065.

27. B.R. Sivertsen et al., Journal of Power Sources 141 (2005) 65-78, doi:10.1016/j.jpowsour.2004.08.054 